International Journal of Mathematics (to appear).

\title{
CLASSIFICATION OF RICCI SOLITONS ON EUCLIDEAN HYPERSURFACES
}

\author{
BANG-YEN CHEN AND SHARIEF DESHMUKH
}

\begin{abstract}
A Ricci soliton $(M, g, v, \lambda)$ on a Riemannian manifold $(M, g)$ is said to have concurrent potential field if its potential field $v$ is a concurrent vector field. Ricci solitons arisen from concurrent vector fields on Riemannian manifolds were studied recently in $[9$. The most important concurrent vector field is the position vector field on Euclidean submanifolds. In this paper we completely classify Ricci solitons on Euclidean hypersurfaces arisen from the position vector field of the hypersurfaces.
\end{abstract}

\section{INTRODUCTION}

A smooth vector field $\xi$ on a Riemannian manifold $(M, g)$ is said to define a Ricci soliton if it satisfies

$$
\frac{1}{2} \mathcal{L}_{\xi} g+R i c=\lambda g
$$

where $\mathcal{L}_{\xi} g$ is the Lie-derivative of the metric tensor $g$ with respect to $\xi, R i c$ is the Ricci tensor of $(M, g)$ and $\lambda$ is a constant. Compact Ricci solitons are the fixed points of the Ricci flow: $\frac{\partial g(t)}{\partial t}=-2 \operatorname{Ric}(g(t))$ projected from the space of metrics onto its quotient modulo diffeomorphisms and scalings, and often arise as blowup limits for the Ricci flow on compact manifolds. Further, Ricci solitons model the formation of singularities in the Ricci flow and they correspond to self-similar solutions (cf. [19]).

We shall denote a Ricci soliton by $(M, g, \xi, \lambda)$. We call the vector field $\xi$ the potential field. A Ricci soliton $(M, g, \xi, \lambda)$ is called shrinking, steady or expanding according to $\lambda>0, \lambda=0$, or $\lambda<0$, respectively. A trivial Ricci soliton is one for which $\xi$ is zero or Killing, in which case the metric is Einsteinian.

A Ricci soliton $(M, g, \xi, \lambda)$ is called a gradient Ricci soliton if its potential field $\xi$ is the gradient of some smooth function $f$ on $M$. We shall denote a gradient Ricci soliton by $(M, g, f, \lambda)$ and call the smooth function $f$ the potential function. A gradient Ricci soliton $(M, g, f, \lambda)$ is called trivial if its potential function $f$ is a constant. It follows from (1.1) that trivial gradient Ricci solitons are trivial

2000 Mathematics Subject Classification. 53C25, 53C40.

Key words and phrases. Ricci soliton, Einstein manifold, Euclidean hypersurface, position vector field, concurrent vector field. 
Ricci solitons automatically since $\xi=\nabla f$. It is well-known that if $(M, g, \xi, \lambda)$ is a compact Ricci soliton, then the potential field $\xi$ is a gradient of some smooth function $f$ up to the addition of a Killing field (cf. [20]) and thus every compact Ricci soliton is a gradient Ricci soliton.

During the last two decades, the geometry of Ricci solitons has been the focus of attention of many mathematicians. In particular, it has become more important after Grigory Perelman applied Ricci solitons to solve the long standing Poincaré conjecture posed in 1904. G. Perelman observed in 20] that the Ricci solitons on compact simply connected Riemannian manifolds are gradient Ricci solitons as solutions of Ricci flow.

A vector field on a Riemannian manifold $M$ is called concurrent if it satisfies

$$
\nabla_{X} v=X, \quad X \in T M
$$

The best known example of Riemannian manifolds endowed with concurrent vector fields is the Euclidean space with the concurrent vector field given by its position vector field $\mathbf{x}$ (with respect to the origin).

For a submanifold $M^{n}$ of a Euclidean $m$-space $\mathbb{E}^{m}$, the most natural tangent vector field of $M^{n}$ is the tangential component of the position vector field $\mathbf{x}$ of $M^{n}$ in $\mathbb{E}^{m}$ (cf. for instance [4, [5]). Ricci solitons on Euclidean submanifolds arisen from such a vector field were studied recently by the authors in [9]. Several fundamental results in this respect were proved in [9]. We remark that Ricci solitons on submanifolds have also been studied in [11, 12, 13 by J. T. Cho and M. Kimura from a different viewpoint. They proved several interesting results on Ricci solitons on submanifolds; however their potential fields of the Ricci solitons are quite different from ours.

In this paper we completely classify Ricci solitons on Euclidean hypersurfaces whose potential field arisen from the position vector field.

\section{BASIC DEFINITIONS AND FORMULAS}

For general references on Riemannian submanifolds, we refer to [2, 6, 7].

Let $\left(N^{m}, \tilde{g}\right)$ be an $m$-dimensional Riemannian manifold and $\phi: M^{n} \rightarrow N^{m}$ an isometric immersion of a Riemannian $n$-manifold $\left(M^{n}, g\right)$ into $\left(N^{m}, \tilde{g}\right)$. Denote by $\nabla$ and $\tilde{\nabla}$ the Levi-Civita connections on $\left(M^{n}, g\right)$ and $\left(N^{m}, \tilde{g}\right)$, respectively.

For vector fields $X, Y$ tangent to $M^{n}$ and $\eta$ normal to $M^{n}$, the formula of Gauss and the formula of Weingarten are given respectively by

$$
\begin{aligned}
& \tilde{\nabla}_{X} Y=\nabla_{X} Y+h(X, Y), \\
& \tilde{\nabla}_{X} \eta=-A_{\eta} X+D_{X} \eta,
\end{aligned}
$$

where $\nabla_{X} Y$ and $h(X, Y)$ are the tangential and the normal components of $\tilde{\nabla}_{X} Y$. Similarly, $-A_{\eta} X$ and $D_{X} \eta$ are the tangential and normal components of $\tilde{\nabla}_{X} \eta$. 
These two formulas define the second fundamental form $h$, the shape operator $A$, and the normal connection $D$ of $M^{n}$ in the ambient space $N^{m}$.

It is well-known that each $A_{\eta}$ is a self-adjoint endomorphism. The shape operator $A$ and the second fundamental form $h$ are related by

$$
\langle h(X, Y), \eta\rangle=\left\langle A_{\eta} X, Y\right\rangle,
$$

where $\langle$,$\rangle denotes the inner product of M^{n}$ as well as of $N^{m}$.

The mean curvature vector $H$ of $M^{n}$ in $N^{m}$ is defined by

$$
H=\left(\frac{1}{n}\right) \text { trace } h .
$$

A submanifold $M^{n}$ is called minimal if its mean curvature vector field vanishes identically. It is called totally umbilical if the second fundamental form satisfies $h(X, Y)=g(X, Y) H$ for tangent vectors $X, Y$.

The equations of Gauss and Codazzi are given respectively by

$$
\begin{gathered}
\langle R(X, Y) Z, W\rangle=\langle\tilde{R}(X, Y) Z, W\rangle+\langle h(X, W), h(Y, Z)\rangle \\
-\langle h(X, Z), h(Y, W)\rangle, \\
(\tilde{R}(X, Y) Z)^{\perp}=\left(\bar{\nabla}_{X} h\right)(Y, Z)-\left(\bar{\nabla}_{Y} h\right)(X, Z),
\end{gathered}
$$

for vectors $X, Y, Z, W$ tangent to $M$, where $(\tilde{R}(X, Y) Z)^{\perp}$ is the normal component of $\tilde{R}(X, Y) Z$ and $\bar{\nabla} h$ is defined by

$$
\left(\bar{\nabla}_{X} h\right)(Y, Z)=D_{X} h(Y, Z)-h\left(\nabla_{X} Y, Z\right)-h\left(Y, \nabla_{X} Z\right) .
$$

For a function $f$ on $M^{n}$, we denote by $\nabla f$ and $H^{f}$ the gradient of $f$ and the Hessian of $f$, respectively. Thus we have

$$
\begin{aligned}
& g(\nabla f, X)=X f, \\
& H^{f}(X, Y)=X Y f-\left(\nabla_{X} Y\right) f .
\end{aligned}
$$

Throughout this paper, $S^{k}(r)$ denote the $k$-dimensional sphere of radius $r$ and $\mathbb{E}^{k}$ the Euclidean $k$-space.

\section{DOUbly WARPED AND TWISTED PRODUCTS}

For a differential manifold $M$, we denote by $C^{\infty}(M)$ the space of differentiable functions on $M$, and by $T M$ the tangent bundle of $M$.

Let $M_{1}$ and $M_{2}$ be pseudo-Riemannian manifolds with pseudo-Riemannian metrics $g_{1}$ and $g_{2}$, respectively. If $f_{1}$ and $f_{2}$ are positive functions in $C^{\infty}\left(M_{1} \times M_{2}\right)$ and $\pi_{r}: M \rightarrow M_{r}$ denotes the canonical projection for $r=1,2$. Then the doubly twisted product $M_{1} \times_{\left(f_{1}, f_{2}\right)} M_{2}$ of $\left(M_{1}, g_{1}\right)$ and $\left(M_{2}, g_{2}\right)$ is the manifold $M_{1} \times M_{2}$ equipped with the pseudo-Riemannian metric $g$ defined by

$$
g(X, Y)=f_{1}^{2} \cdot g_{1}\left(\pi_{1_{*}} X, \pi_{1_{*}} Y\right)+f_{2}^{2} \cdot g_{2}\left(\pi_{2_{*}}(X), \pi_{2_{*}} Y\right)
$$


for tangent vectors $X, Y \in T\left(M_{1} \times M_{2}\right)$ (cf. [3, 21]). In particular, if either $f_{1}=1$ or $f_{2}=1$, then the doubly twisted product is a twisted product (in the usual sense) (see [3, page 66]).

Definition 3.1. A doubly twisted product $M_{1} \times_{\left(f_{1}, f_{2}\right)} M_{2}$ is called a doubly warped product if $f_{1} \in C^{\infty}\left(M_{2}\right)$ and $f_{2} \in C^{\infty}\left(M_{1}\right)$. In particular, if either $f_{1}=1$ or $f_{2}=1$, then the doubly warped product is a warped product (in the usual sense).

Definition 3.2. A doubly twisted product $M_{1} \times_{\left(f_{1}, f_{2}\right)} M_{2}$ is called a warped-twisted product if $f_{1} \in C^{\infty}\left(M_{2}\right)$ and $f_{2} \in C^{\infty}\left(M_{1} \times M_{2}\right)$. Similarly, $M_{1} \times{ }_{\left(f_{1}, f_{2}\right)} M_{2}$ is a twisted-warped product if $f_{1} \in C^{\infty}\left(M_{1} \times M_{2}\right)$ and $f_{2} \in C^{\infty}\left(M_{1}\right)$.

A foliation $\mathcal{D}$ on a manifold $M$ is an integrable distribution, i.e., $\mathcal{D}$ is a vector subbundle of the tangent bundle $T M$ such that, for any vector fields $X, Y$ in $\mathcal{D}$, the Lie bracket $[X, Y]$ takes values in $\mathcal{D}$ as well. A foliation $\mathcal{D}$ on a pseudo-Riemannian manifold $M$ is called totally umbilical, if every leaf of $\mathcal{D}$ is a totally umbilical submanifold of $M$. If, in addition, the mean curvature vector of every leaf is parallel in the normal bundle, then $\mathcal{D}$ is called a spherical foliation. In this case, leaves of $\mathcal{D}$ are extrinsic spheres of $M$. If leaves of a foliation $\mathcal{D}$ are totally geodesic submanifolds, $\mathcal{D}$ is called a totally geodesic foliation.

The following result was proved in 21 .

Theorem 3.1. Let $g$ be a pseudo-Riemannian metric on $M_{1} \times M_{2}$. If the canonical foliations $\mathcal{D}_{1}$ and $\mathcal{D}_{2}$ intersect perpendicularly everywhere, then $g$ is the metric of

(a) a double-twisted product $M_{1} \times_{\left(f_{1}, f_{2}\right)} M_{2}$ if and only if $\mathcal{D}_{1}$ and $\mathcal{D}_{2}$ are totally umbilical foliations;

(b) a twisted product $M_{1} \times_{f} M_{2}$ if and only if $\mathcal{D}_{1}$ is a totally geodesic and $\mathcal{D}_{2}$ a totally umbilical foliation;

(c) a warped product $M_{1} \times_{f} M_{2}$ if and only if $\mathcal{D}_{1}$ is a totally geodesic and $\mathcal{D}_{2}$ a spherical foliation.

For the proof of our main result, we need the following.

Theorem 3.2. Let $g$ be a pseudo-Riemannian metric on $M_{1} \times M_{2}$. If the canonical foliations $\mathcal{D}_{1}$ and $\mathcal{D}_{2}$ intersect perpendicularly everywhere, then we have:

(1) If $\mathcal{D}_{1}$ is a totally umbilical foliation and $\mathcal{D}_{2}$ a spherical foliation, then the metric $g$ on $M_{1} \times M_{2}$ is a twisted-warped product;

(2) If $\mathcal{D}_{1}$ is a spherical foliation and $\mathcal{D}_{2}$ a totally umbilical foliation, then $g$ is a warped-twisted product;

(3) If $\mathcal{D}_{1}$ and $\mathcal{D}_{2}$ are spherical foliations, then $g$ is a doubly warped product.

Proof. Let $g$ be a pseudo-Riemannian metric on $M_{1} \times M_{2}$ such that their canonical foliations $\mathcal{D}_{1}$ and $\mathcal{D}_{2}$ intersect perpendicularly everywhere. If $\mathcal{D}_{1}$ and $\mathcal{D}_{2}$ are totally umbilical foliations, Theorem 3.1(1) implies that $g$ is a doubly twisted product 
metric. So the metric $g$ can be expressed as (3.1). Hence we have

$$
g(X, Z)=0, \quad X \in \mathfrak{X}\left(M_{1}\right), \quad Z \in \mathfrak{X}\left(M_{2}\right),
$$

where $\mathfrak{X}(N)$ consists of vector fields of a manifold $N$.

Since $[X, Z]=0$ for $X \in \mathfrak{X}\left(M_{1}\right)$ and $Z \in \mathfrak{X}\left(M_{2}\right)$, we get

$$
\nabla_{X} Z=\nabla_{Z} X \text {. }
$$

Therefore, for $X \in \mathfrak{X}\left(M_{1}\right)$ and $Z, W \in \mathfrak{X}\left(M_{2}\right)$, we obtain

$$
X g(Z, W)=X\left(f_{2}^{2} \cdot g_{2}(Z, W)\right)=\frac{2 X f_{2}}{f_{2}} g(Z, W) .
$$

On the other hand, by using (3.3) and $g([Z, W], X)=0$ we find

$$
\begin{aligned}
X g(Z, W) & =g\left(\nabla_{Z} X, W\right)+g\left(Z, \nabla_{W} X\right) \\
& =-g\left(X, \nabla_{Z} W\right)-g\left(\nabla_{W} Z, X\right) \\
& =-2 g\left(X, \nabla_{Z} W\right) .
\end{aligned}
$$

Hence it follows from (3.4) and (3.5) that the second fundamental form $h_{2}$ of $M_{2}$ in $M_{1} \times_{\left(f_{1}, f_{2}\right)} M_{2}$ is given by

$$
h_{2}(Z, W)=-\frac{\nabla^{1} f_{2}}{f_{2}} g(Z, W),
$$

where $\nabla^{1} f_{2}$ is defined by

$$
\nabla^{1} f_{2}=\sum_{i=1}^{p}\left(E_{i} f_{2}\right) E_{i}, \quad p=\operatorname{dim} M_{1}
$$

and $E_{1}, \ldots, E_{p}$ is an orthonormal basis of $T M_{1}$. In particular, if we choose $E_{1}$ in the direction of $\nabla^{1} f_{2}$, then (3.7) reduces to

$$
\nabla^{1} f_{2}=\left(E_{1} f_{2}\right) E_{1} .
$$

Hence it follows from (3.6) and (3.8) that the mean curvature vector $H_{2}$ of $M_{2}$ in $M_{1} \times{ }_{\left(f_{1}, f_{2}\right)} M_{2}$ is given by

$$
H_{2}=-\left(E_{1} \ln f_{2}\right) E_{1} .
$$

Thus we have

$$
\nabla_{Z} H_{2}=-Z\left(E_{1} \ln f_{2}\right) E_{1}-\left(E_{1} \ln f_{2}\right) \nabla_{Z} E_{1}, \quad \forall Z \in T M_{2} .
$$

Therefore the normal connection $D^{2}$ of $M_{2}$ in $M_{1} \times_{\left(f_{1}, f_{2}\right)} M_{2}$ satisfies

$$
D_{Z}^{2} H_{2}=-Z\left(E_{1} \ln f_{2}\right) E_{1}-\left(E_{1} \ln f_{2}\right) D_{Z}^{2} E_{1} .
$$

Hence if $H_{2}$ is parallel in the normal bundle of $M_{2}$ in $M_{1} \times{ }_{\left(f_{1}, f_{2}\right)} M_{2}$, then we obtain $X\left(Z \ln f_{2}\right)=Z\left(X \ln f_{2}\right)=0$ for $X \in \mathfrak{X}\left(M_{1}\right)$ and $Z \in \mathfrak{X}\left(M_{2}\right)$. Consequently, if $H_{2}$ is parallel in the normal bundle, then $f_{2} \in C^{\infty}\left(M_{1}\right)$. Therefore the doubly twisted product $M_{1} \times_{\left(f_{1}, f_{2}\right)} M_{2}$ is a twisted-warped product. This gives statement (1).

Statements (2) and (3) can be proved in the same way as statement (1). 


\section{Some PREliminary ReSUlts on Ricci solitons}

We make the following

Assumption. $\left(N^{m}, \tilde{g}\right)$ is a Riemannian m-manifold endowed with a concurrent vector field $v$. For a submanifold $M^{n}$ of $N^{m}, v^{T}$ and $v^{\perp}$ denote the tangential and normal components of $v$ on $M^{n}$, respectively.

The following two results were proved in 9 .

Theorem 4.1. A submanifold $M^{n}$ in $N^{m}$ admits a Ricci soliton $\left(M^{n}, g, v^{T}, \lambda\right)$ if and only if the Ricci tensor of $\left(M^{n}, g\right)$ satisfies

$$
\operatorname{Ric}(X, Y)=(\lambda-1) g(X, Y)-\left\langle h(X, Y), v^{\perp}\right\rangle
$$

for any $X, Y$ tangent to $M^{n}$.

Proposition 4.1. If $\left(M^{n}, g, \mathbf{x}^{T}, \lambda\right)$ is a Ricci soliton on a hypersurface of $M^{n}$ of $\mathbb{E}^{n+1}$, then $M^{n}$ has at most two distinct principal curvatures given by

$$
\kappa_{1}, \kappa_{2}=\frac{n \alpha+\rho \pm \sqrt{(n \alpha+\rho)^{2}+4-4 \lambda}}{2}
$$

where $\alpha$ is the mean curvature and $\rho$ is the support function of $M^{n}$, i.e., $\rho=\langle\mathbf{x}, N\rangle$ and $H=\alpha N$ with $N$ being a unit normal vector field.

The following theorem was proved in [9].

Theorem 4.2. Let $\left(M^{n}, g, \mathbf{x}^{T}, \lambda\right)$ be a shrinking Ricci soliton on a hypersurface of $M^{n}$ of $\mathbb{E}^{n+1}$ with $\lambda=1$. Then $M^{n}$ is an open portion of one of the following hypersurfaces of $\mathbb{E}^{n+1}$ :

(1) A hyperplane through the origin o.

(2) A hypersphere centered at the origin.

(3) A flat hypersurface generated by lines through the origin of $\mathbb{E}^{n+1}$.

(4) A spherical hypercylinder $S^{k}(\sqrt{k-1}) \times \mathbb{E}^{n-k}, 2 \leq k \leq n-1$.

We also need the following lemma.

Lemma 4.1. Let $M^{n}$ be a rotational hypersurface of $\mathbb{E}^{n+1}$ given by

$$
\begin{gathered}
\mathbf{x}\left(x_{1}, \ldots, x_{n}\right)=\left(x_{1}, f\left(x_{1}\right) \sin x_{2}, f\left(x_{1}\right) \cos x_{2} \sin x_{3}, \ldots,\right. \\
\left.f\left(x_{1}\right) \cos x_{2} \cdots \cos x_{n-1} \sin x_{n}, f\left(x_{1}\right) \cos x_{2} \cdots \cos x_{n}\right) .
\end{gathered}
$$

If $\left(M^{n}, g, \mathbf{x}^{T}, \lambda\right)$ is a Ricci soliton, then $M^{n}$ is an open portion of a hypersphere.

Proof. It is easy to verify from (4.3) that the metric tensor of $M^{n}$ is given by

$$
g=\left(1+f^{\prime}\left(x_{1}\right)^{2}\right) d x_{1}^{2}+f^{2}\left(x_{1}\right)\left\{d x_{2}^{2}+\cos ^{2} x_{2} d x_{3}^{2}+\cdots+\prod_{j=2}^{n-1} \cos ^{2} x_{j} d x_{n}^{2}\right\} .
$$


A direct computation shows that the Ricci tensor and the second fundamental form of $M^{n}$ satisfy

$$
\begin{aligned}
& \operatorname{Ric}\left(\partial_{x_{1}}, \partial_{x_{1}}\right)=\frac{-2 f^{\prime \prime}}{f\left(1+f^{\prime 2}\right)}, \quad \operatorname{Ric}\left(\partial_{x_{2}}, \partial_{x_{2}}\right)=\frac{1+f^{\prime 2}-f f^{\prime \prime}}{\left(1+f^{\prime 2}\right)^{2}} \\
& \left\langle h\left(\partial_{x_{1}}, \partial_{x_{1}}\right), \mathbf{x}^{\perp}\right\rangle=\frac{\left(f-x_{1} f^{\prime}\right) f^{\prime \prime}}{1+f^{\prime 2}}, \quad\left\langle h\left(\partial_{x_{2}}, \partial_{x_{2}}\right), \mathbf{x}^{\perp}\right\rangle=\frac{\left(x_{1} f^{\prime}-f\right) f}{1+f^{\prime 2}} .
\end{aligned}
$$

where $\mathbf{x}^{\perp}$ is the normal component of the position vector field $\mathbf{x}$ of $M^{n}$ in $\mathbb{E}^{n+1}$.

If $\left(M^{n}, g, \mathbf{x}^{T}, \lambda\right)$ is a Ricci soliton, then Theorem 4.1 implies that

$$
\frac{\operatorname{Ric}\left(\partial_{x_{1}}, \partial_{x_{1}}\right)+\left\langle h\left(\partial_{x_{1}}, \partial_{x_{1}}\right), \mathbf{x}^{\perp}\right\rangle}{g_{11}}=\frac{\operatorname{Ric}\left(\partial_{x_{2}}, \partial_{x_{2}}\right)+\left\langle h\left(\partial_{x_{2}}, \partial_{x_{2}}\right), \mathbf{x}^{\perp}\right\rangle}{g_{22}} .
$$

By applying (4.4)-(4.7), we obtain

(i) $1-f^{2}+x_{1} f f^{\prime}=0$ or

(ii) $1+f^{\prime 2}+f f^{\prime \prime}=0$.

Case (i): $1-f^{2}+x_{1} f f^{\prime}=0$. In this case, we obtain $f\left(x_{1}\right)= \pm \sqrt{1+b^{2} x_{1}^{2}}$ for some constant $b \neq 0$. Hence

$$
\frac{\operatorname{Ric}\left(\partial_{x_{1}}, \partial_{x_{1}}\right)-\left\langle h\left(\partial_{x_{1}}, \partial_{x_{1}}\right), \mathbf{x}^{\perp}\right\rangle}{g_{11}}=\frac{-b^{2}}{\left(1+b^{2} x_{1}^{2}\left(1+b^{2}\right)\right)^{2}},
$$

is non-constant. Therefore $\left(M^{n}, g, \mathbf{x}^{T}, \lambda\right)$ cannot be a Ricci soliton.

Case (ii): $1+f^{\prime 2}+f f_{1}^{\prime \prime}=0$. In this case, we have $f(x)= \pm \sqrt{b^{2}-\left(x_{1}+c\right)^{2}}$ for some constant $b, c$. Hence

$$
\frac{\operatorname{Ric}\left(\partial_{x_{i}}, \partial_{x_{i}}\right)-\left\langle h\left(\partial_{x_{i}}, \partial_{x_{i}}\right), \mathbf{x}^{\perp}\right\rangle}{g_{11}}=\frac{2-b^{2}+c^{2}-c x_{1}}{b^{2}}, i=1, \ldots, n,
$$

which is a constant if and only if $c=0$. When $c=0, M^{n}$ is an open portion of a hypersphere, which is obviously a Ricci soliton.

\section{Ricci SOLITONS ON HYPERSURFACES With CONSTANT MEAN CURVATURE}

First, we provide examples of Ricci solitons on Euclidean hypersurfaces with constant mean curvature.

Example 5.1. Let $k$ be a natural number such that $1 \leq k \leq n-1$. Consider the spherical hypercylinder $\phi: S^{k}(r) \times \mathbb{E}^{n-k} \rightarrow \mathbb{E}^{n+1}$ defined by

$$
\left\{\left(\mathbf{y}, x_{k+2}, \ldots, x_{n+1}\right) \in \mathbb{E}^{n+1}: \mathbf{y} \in \mathbb{E}^{k+1} \text { and }\langle\mathbf{y}, \mathbf{y}\rangle=r^{2}\right\} .
$$

It is direct to verify that the spherical hypercylinder $S^{k}(\sqrt{k-1}) \times \mathbb{E}^{n-k}$ in $\mathbb{E}^{n+1}$ satisfies (4.1) for $\lambda=1$ whenever $k \geq 2$. Hence $\left(S^{k}(\sqrt{k-1}) \times \mathbb{E}^{n-k}, g, \mathbf{x}^{T}, \lambda\right)$ with $k \geq 2$ is a shrinking Ricci soliton with $\lambda=1$. Similarly, for any $r>0$, the circular hypercylinder $S^{1}(r) \times \mathbb{E}^{n-1} \subset \mathbb{E}^{n+1}$ is also a trivial Ricci soliton. Obviously, such hypercylinders have constant mean curvature. 
Now, we provide the following classification of Ricci solitons on Euclidean hypersurfaces with constant mean curvature.

Theorem 5.1. Let $\left(M^{n}, g, \mathbf{x}^{T}, \lambda\right)$ be a Ricci soliton on a hypersurface $M^{n}$ of $\mathbb{E}^{n+1}$. If $M^{n}$ has constant mean curvature, then it is one of the following hypersurfaces:

(a) A hyperplane through the origin o.

(b) A hypersphere centered at the origin.

(c) An open part of a circular hypercylinder $S^{1}(r) \times \mathbb{E}^{n-1}, r>0$.

(d) An open part of a spherical hypercylinder $S^{k}(\sqrt{k-1}) \times \mathbb{E}^{n-k}, 2 \leq k \leq n-1$.

Proof. Assume that $\left(M^{n}, g, \mathbf{x}^{T}, \lambda\right)$ is a Ricci soliton on a hypersurface $M^{n}$ of $\mathbb{E}^{n+1}$. Then it follows from Proposition 4.1 that $M^{n}$ has at most two distinct principal curvatures. If $M^{n}$ has only one principal curvature, then it is totally umbilical. In this case we obtain either case (a) or case (b).

If $M^{n}$ has two distinct principal curvatures, then Proposition 4.1 implies that the two principal curvatures are given respectively by

$$
\begin{gathered}
\kappa_{1}=\frac{n \alpha+\rho+\sqrt{(n \alpha+\rho)^{2}+4-4 \lambda}}{2}, \\
\kappa_{2}=\frac{n \alpha+\rho-\sqrt{(n \alpha+\rho)^{2}+4-4 \lambda}}{2} .
\end{gathered}
$$

Let us assume that the multiplicities of $\kappa_{1}$ and $\kappa_{2}$ are $p$ and $n-p$, respectively. Then we find from (5.1) that

$$
(2-n) n \alpha=n \rho+(2 p-n) \sqrt{(n \alpha+\rho)^{2}+4-4 \lambda} .
$$

Suppose that $M^{n}$ has constant mean curvature $\alpha$. Then it follows from (5.2) that the support function $\rho=\langle\mathbf{x}, N\rangle$ is constant. Thus we have

$$
0=X \rho=-\left\langle\mathbf{x}, A_{N} X\right\rangle=-\left\langle h\left(X, \mathbf{x}^{T}\right), N\right\rangle .
$$

If $\mathbf{x}^{T} \neq 0$, then (5.3) implies that one of $\kappa_{1}, \kappa_{2}$ is zero. So we obtain $\lambda=1$. In this case, we obtain case (c) or case (d) by Theorem 4.2

If $\mathbf{x}^{T}=0$, then $\mathbf{x}$ is normal to $M^{n}$. Thus $\langle\mathbf{x}, \mathbf{x}\rangle$ must be a constant. Therefore in this case we obtain case (b) of the theorem.

\section{Classification of Ricci solitons on Euclidean hypersurfaces}

The main purpose of this paper is to prove the following theorem which classifies Ricci solitons on Euclidean hypersurfaces arisen from the position vector field.

Theorem 6.1. Let $\left(M^{n}, g, \mathbf{x}^{T}, \lambda\right)$ be a Ricci soliton on a hypersurface of $M^{n}$ of $\mathbb{E}^{n+1}$. Then $M^{n}$ is one of the following hypersurfaces of $\mathbb{E}^{n+1}$ :

(1) A hyperplane through the origin 0.

(2) A hypersphere centered at the origin.

(3) An open part of a flat hypersurface generated by lines through the origin o;

(4) An open part of a circular hypercylinder $S^{1}(r) \times \mathbb{E}^{n-1}, r>0$; 
(5) An open part of a spherical hypercylinder $S^{k}(\sqrt{k-1}) \times \mathbb{E}^{n-k}, 2 \leq k \leq n-1$.

Proof. Let $\left(M^{n}, g, \mathbf{x}^{T}, \lambda\right)$ be a Ricci soliton on a Euclidean hypersurface $M^{n}$. It follows from Proposition 4.1 that $M^{n}$ has at most two distinct principal curvatures. If $M^{n}$ has only one principal curvature, then it is totally umbilical. Thus we obtain case (1) or case (2) of the theorem. Hence, from now on we may assume that $M^{n}$ has two distinct principal curvatures $\kappa_{1}, \kappa_{2}$. Let us assume that their multiplicities are $m\left(\kappa_{1}\right)=p$ and $m\left(\kappa_{2}\right)=n-p$. Hence we find from (5.1) that

$$
n \alpha=\frac{n}{2}(n \alpha+\rho)+\frac{2 p-n}{2} \sqrt{(n \alpha+\rho)^{2}+4-4 \lambda} .
$$

Case (1): $\lambda=1$. In this case, the theorem follows from Theorem 4.2 ,

Case (2): $\lambda \neq 1$. Proposition 4.1 implies $\kappa_{1}, \kappa_{2} \neq 0$. Without loss of generality, we may put

$$
\begin{gathered}
h\left(e_{i}, e_{j}\right)=\delta_{i j} \kappa_{1} N, h\left(e_{i}, e_{\beta}\right)=0, h\left(e_{\beta}, e_{\gamma}\right)=\delta_{\beta \gamma} \kappa_{2} N, \\
i, j=1, \ldots, p ; \beta, \gamma=p+1, \ldots, n,
\end{gathered}
$$

with respect to an orthonormal tangent frame $\left\{e_{1}, \ldots, e_{n}\right\}$ of $M^{n}$, where $\delta_{i j}, \delta_{\beta \gamma}$ are Kronecker deltas, and $N$ is a unit normal vector field. Define the connection forms $\omega_{i}^{j}(i, j=1, \ldots, n)$ on $M^{n}$ by

$$
\nabla_{X} e_{A}=\sum_{B=1}^{n} \omega_{A}^{B}(X) e_{B}, \quad A=1, \ldots, n .
$$

We define two distributions $\mathcal{D}_{1}, \mathcal{D}_{2}$ by

$$
\mathcal{D}_{1}=\operatorname{Span}\left\{e_{1}, \ldots, e_{p}\right\}, \quad \mathcal{D}_{2}=\operatorname{Span}\left\{e_{p+1}, \ldots, e_{n}\right\} .
$$

Case (2.1): $2 \leq p \leq n-2$. In this case, both $\kappa_{1}$ and $\kappa_{2}$ has multiplicity at least 2 . So we may derive from (2.7), (6.2) and the following equations

$$
\left(\bar{\nabla}_{e_{i}} h\right)\left(e_{j}, e_{j}\right)=\left(\bar{\nabla}_{e_{j}} h\right)\left(e_{i}, e_{j}\right), \quad 1 \leq i \neq j \leq p
$$

of Codazzi that $e_{i} \kappa_{1}=0$ for $i=1, \ldots, p$. Thus $\nabla \kappa_{1} \in \mathcal{D}_{2}$. Similarly, we also have $\nabla \kappa_{2} \in \mathcal{D}_{1}$. Thus we can choose $e_{1}, \ldots, e_{n}$ in such way that

$$
\nabla \kappa_{1}=\varphi_{1} e_{n}, \quad \nabla \kappa_{2}=\varphi_{2} e_{1}
$$

for some functions $\varphi_{1}, \varphi_{2}$. Also, it follows from (2.7), (6.2) and

$$
\left(\bar{\nabla}_{e_{\beta}} h\right)\left(e_{i}, e_{j}\right)=\left(\bar{\nabla}_{e_{j}} h\right)\left(e_{i}, e_{\beta}\right), \quad 1 \leq i, j \leq p ; p+1 \leq \beta \leq n,
$$

that

$$
\omega_{i}^{\beta}\left(e_{j}\right)=\delta_{i j}\left(\frac{e_{\beta} \kappa_{1}}{\kappa_{1}-\kappa_{2}}\right), i, j=1, \ldots, p ; \beta=p+1, \ldots, n .
$$

Since $e_{i} \kappa_{1}=e_{\beta} \kappa_{2}=0$, we derive from (6.6) that

$$
\omega_{i}^{\beta}\left(e_{j}\right)=\delta_{i j} e_{\beta}\left(\ln \left|\kappa_{1}-\kappa_{2}\right|\right), \quad i, j=1, \ldots, p ; \beta=p+1, \ldots, n
$$


Similarly, we also have

$$
\omega_{\beta}^{j}\left(e_{\gamma}\right)=\delta_{\beta \gamma} e_{j}\left(\ln \left|\kappa_{1}-\kappa_{2}\right|\right), \quad j=1, \ldots, p ; \beta, \gamma=p+1, \ldots, n .
$$

Consequently, (6.5), (6.7) and (6.8) give

$$
\begin{aligned}
& \omega_{i}^{\beta}\left(e_{j}\right)=\delta_{i j} \delta_{\beta n} e_{n}\left(\ln \left|\kappa_{1}-\kappa_{2}\right|\right), \\
& \omega_{\beta}^{j}\left(e_{\gamma}\right)=\delta_{1 j} \delta_{\beta \gamma} e_{1}\left(\ln \left|\kappa_{1}-\kappa_{2}\right|\right),
\end{aligned}
$$

for $i, j=1, \ldots, p ; \beta, \gamma=p+1, \ldots, n$. From (6.6) we obtain $\left\langle\left[e_{i}, e_{j}\right], e_{\beta}\right\rangle=0$ for $1 \leq i, j \leq p ; p+1 \leq \beta \leq n$. Hence $\mathcal{D}_{1}$ is an integrable distribution. Similarly, $\mathcal{D}_{2}$ is also integrable.

Let $L_{1}^{p}$ be a leaf of $\mathcal{D}_{1}$ and $L_{2}^{n-p}$ a leaf of $\mathcal{D}_{2}$. Then it follows from (6.7) and (6.8) that $L_{1}^{p}$ and $L_{2}^{n-p}$ are totally umbilical submanifolds of $M^{n}$. Thus Theorem 3.1 implies that $M^{n}$ is a doubly twisted product $L_{1}^{p} \times_{\left(f_{1}, f_{2}\right)} L_{2}^{n-p}$ whose metric tensor is given by

$$
g=f_{1}^{2} g_{1}+f_{2}^{2} g_{2},
$$

where $g_{1}$ and $g_{2}$ are the metric tensors of $L_{1}^{p}$ and $L_{2}^{n-p}$, respectively.

It follows from (6.9) and (6.10) that the mean curvature vectors $\stackrel{\circ}{H}_{1}$ and $\stackrel{\circ}{H}_{2}$ of $L_{1}^{p}$ and $L_{2}^{n-p}$ in $M^{n}$ are given respectively by

$$
\begin{aligned}
& \stackrel{\circ}{H}_{1}=\left\{e_{n}\left(\ln \left|\kappa_{1}-\kappa_{2}\right|\right)\right\} e_{n}, \\
& \stackrel{\circ}{H}_{2}=\left\{e_{1}\left(\ln \left|\kappa_{1}-\kappa_{2}\right|\right)\right\} e_{1},
\end{aligned}
$$

Therefore (6.2) and (6.12) show that the mean curvature vectors $\hat{H}_{1}$ and $\hat{H}_{2}$ of $L_{1}^{p}$ and $L_{2}^{n-p}$ in $\mathbb{E}^{n+1}$ are given respectively by

$$
\hat{H}_{1}=\stackrel{\circ}{H}_{1}+\kappa_{1} N, \quad \hat{H}_{2}=\stackrel{\circ}{H}_{2}+\kappa_{2} N .
$$

Since $L_{1}^{p}$ and $L_{2}^{n-p}$ are totally umbilical in $\mathbb{E}^{n+1}$, both $\hat{H}_{1}$ and $\hat{H}_{2}$ are parallel in their normal bundles in $\mathbb{E}^{n+1}$ (cf. [2]). Hence the normal connection $\hat{D}^{1}$ of $L_{1}^{p}$ and $\hat{D}^{2}$ of $L_{2}^{n-p}$ in $\mathbb{E}^{n+1}$ satisfy

$$
0=\hat{D}_{X}^{1} \hat{H}_{1}=\hat{D}_{Z}^{2} \hat{H}_{2}
$$

for any $X \in T L_{1}^{p}$ and $Z \in T L_{2}^{n-p}$. Thus, after applying Weingarten's formula of $L_{1}^{p}$ in $\mathbb{E}^{n+1}$, we find

$$
\tilde{\nabla}_{e_{i}} \hat{H}_{1}=-\hat{A}_{\hat{H}_{1}}^{1} e_{i}=\psi_{1} e_{i}
$$

for some function $\psi_{1}$ on $L_{1}^{p}$, where $\hat{A}^{1}$ denotes the shape operator of $L_{1}^{p}$ in $\mathbb{E}^{n+1}$. Hence we have

$$
\begin{aligned}
& \psi_{1} e_{i}=\tilde{\nabla}_{e_{i}} \hat{H}_{1}=\tilde{\nabla}_{e_{i}} \stackrel{\circ}{H}_{1}+\tilde{\nabla}_{e_{i}}\left(\kappa_{1} N\right) \\
& =\nabla_{e_{i}} \stackrel{\circ}{H}_{1}+h\left(e_{i}, \stackrel{\circ}{H}_{1}\right)-\kappa_{1} A_{N} e_{i}+D_{e_{i}}\left(\kappa_{1} N\right) \\
& =-{\stackrel{\circ}{H_{H}}}_{\stackrel{\circ}{1}_{1}}^{1} e_{i}+\stackrel{\circ}{D}_{e_{i}}^{1} \stackrel{\circ}{H}_{1}+h\left(e_{i}, \stackrel{\circ}{H}_{1}\right)-\kappa_{1}^{2} e_{i}+\left(e_{i} \kappa_{1}\right) N
\end{aligned}
$$


for $i=1, \ldots, p$, where $\AA^{1}$ and $\stackrel{\circ}{D}^{1}$ are the shape operator and the normal connection of $L_{1}^{p}$ in $M^{n}$, respectively. It follows from (6.16) that $\stackrel{\circ}{D}_{e_{i}}^{1} \stackrel{\circ}{H}_{1}=0$. Thus the mean curvature vector $H_{1}^{\circ}$ of $L_{1}^{p}$ in $M^{n}$ is parallel in the normal bundle. Hence $L_{1}^{p}$ is an extrinsic sphere in $M^{n}$. Therefore $\mathcal{D}_{1}$ is a spherical distribution in $M^{n}$. Similarly, $\mathcal{D}_{2}$ is also a spherical distribution in $M^{n}$. Consequently, by Theorem 3.2 $L_{1}^{p} \times_{\left(f_{1}, f_{2}\right)} L_{2}^{n-p}$ is a doubly warped product whose metric tensor takes the form:

$$
g=F^{2} g_{L_{1}}+G^{2} g_{L_{2}}
$$

where $F \in C^{\infty}\left(L_{2}^{n-p}\right), G \in C^{\infty}\left(L_{1}^{p}\right)$. Moreover, since $L_{1}^{p}, L_{2}^{n-p}$ are non-totally geodesic totally umbilical submanifolds of $\mathbb{E}^{n+1}$, we may assume $L_{1}^{p}=S^{p}(1)$ and $L_{2}^{n-p}=S^{n-p}(1)$ locally. Hence $M^{n}$ is locally the doubly warped product $S^{p}(1) \times_{(F, G)} S^{n-p}(1)$. Thus, if we choose $\left\{u_{1}, \ldots, u_{p}\right\}$ and $\left\{v_{p+1}, \ldots, v_{n}\right\}$ to be isothermal coordinate systems of $S^{p}(1)$ and $S^{n-p}(1)$, respectively, then we obtain

$$
g=F^{2} U^{2} \sum_{j=1}^{p} d u_{j}^{2}+G^{2} V^{2} \sum_{\gamma=p+1}^{n} d v_{\gamma}^{2},
$$

where $F=F\left(v_{p+1}, \ldots, v_{n}\right), G=G\left(u_{1}, \ldots, u_{p}\right)$, and

$$
U=\frac{2}{1+\sum_{i=1}^{p} u_{i}^{2}}, \quad V=\frac{2}{1+\sum_{\beta=p+1}^{n} v_{\beta}^{2}} .
$$

Put

$$
\partial_{u_{i}}=\frac{\partial}{\partial u_{i}}, \partial_{v_{\beta}}=\frac{\partial}{\partial v_{\beta}}, G_{i}=\frac{\partial G}{\partial u_{i}}, F_{\beta}=\frac{\partial F}{\partial v_{\beta}} .
$$

It follows from (6.18), (6.19) and a direct computation that the Levi-Civita connection $\nabla$ of $\left(M^{n}, g\right)$ satisfies

$$
\begin{aligned}
& \nabla_{\partial_{u_{i}}} \partial_{u_{i}}=-U u_{i} \partial_{u_{i}}+U \sum_{j \neq i} u_{j} \partial_{u_{j}}-\frac{U^{2} F}{V^{2} G^{2}} \sum_{\beta=p+1}^{n} F_{\beta} \partial_{v_{\beta}}, \quad i=1, \ldots, p ; \\
& \nabla_{\partial_{u_{i}}} \partial_{u_{j}}=-U u_{j} \partial_{u_{i}}-U u_{i} \partial_{u_{j}}, \quad 1 \leq i \neq j \leq p \\
& \nabla_{\partial_{u_{i}}} \partial_{v_{\beta}}=\frac{F_{\beta}}{F} \partial_{u_{i}}+\frac{G_{i}}{G} \partial_{v_{\beta}}, \quad i=1, \ldots, p ; \quad \beta=p+1, \ldots, n ; \\
& \nabla_{\partial_{v_{\beta}}} \partial_{v_{\beta}}=-V v_{\beta} \partial_{v_{\beta}}+V \sum_{\gamma \neq \beta} v_{\gamma} \partial_{v_{\gamma}}-\frac{V^{2} G}{U^{2} F^{2}} \sum_{i=1}^{p} G_{i} \partial_{u_{i}}, \quad \beta=p+1, \ldots, n ; \\
& \nabla_{\partial_{v_{\beta}}} \partial_{v_{\gamma}}=-V v_{\gamma} \partial_{v_{\beta}}-V v_{\beta} \partial_{v_{\gamma}}, \quad p+1 \leq \beta \neq \gamma \leq n .
\end{aligned}
$$

By applying (6.20) we find

$$
g\left(R\left(\partial_{u_{i}}, \partial_{u_{j}}\right) \partial_{u_{j}}, \partial_{v_{\beta}}\right)=\frac{U^{2} F}{G} G_{i} F_{\beta}, \quad 1 \leq i \neq j \leq p ; \beta=p+1, \ldots, n .
$$

Also, it follows from (6.2) and Gauss' equation that $g\left(R\left(\partial_{u_{i}}, \partial_{u_{j}}\right) \partial_{u_{j}}, \partial_{v_{\beta}}\right)=0$. By comparing this with (6.21) we get $G_{i} F_{\beta}=0$ for $i=1, \ldots, p ; \beta=p+1, \ldots, n$. Thus, either $F$ or $G$ is a nonzero constant. Without loss of generality, we may assume 
that $F$ is a nonzero constant. So, after applying a suitable dilation we get $F=1$. Hence $g$ is an ordinary warped product, i.e.,

$$
g=U^{2} \sum_{j=1}^{p} d u_{j}^{2}+G^{2} V^{2} \sum_{\gamma=p+1}^{n} d v_{\gamma}^{2} .
$$

Consequently, (6.20) reduces to

$$
\begin{aligned}
& \nabla_{\partial_{u_{i}}} \partial_{u_{i}}=-U u_{i} \partial_{u_{i}}+U \sum_{j \neq i} u_{j} \partial_{u_{j}}, \quad i=1, \ldots, p ; \\
& \nabla_{\partial_{u_{i}}} \partial_{u_{j}}=-U u_{j} \partial_{u_{i}}-U u_{i} \partial_{u_{j}}, \quad 1 \leq i \neq j \leq p, \\
& \nabla_{\partial_{u_{i}}} \partial_{v_{\beta}}=\frac{G_{i}}{G} \partial_{v_{\beta}}, \quad i=1, \ldots, p ; \quad \beta=p+1, \ldots, n ; \\
& \nabla_{\partial_{v_{\beta}}} \partial_{v_{\beta}}=-V v_{\beta} \partial_{v_{\beta}}+V \sum_{\gamma \neq \beta} v_{\gamma} \partial_{v_{\gamma}}-\frac{V^{2} G}{U^{2}} \sum_{i=1}^{p} G_{i} \partial_{u_{i}}, \quad \beta=p+1, \ldots, n ; \\
& \nabla_{\partial_{v_{\beta}}} \partial_{v_{\gamma}}=-V v_{\gamma} \partial_{v_{\beta}}-V v_{\beta} \partial_{v_{\gamma}}, \quad p+1 \leq \beta \neq \gamma \leq n .
\end{aligned}
$$

Therefore, after applying (6.2), (6.23) and Gauss' equation, we may derive that

$$
\begin{aligned}
& \kappa_{1}^{2}=K\left(\partial_{u_{i}}, \partial_{u_{j}}\right)=1, \\
& \kappa_{1} \kappa_{2}=K\left(\partial_{u_{i}}, \partial_{v_{\beta}}\right)=-\frac{H^{G}\left(\partial_{u_{i}}, \partial_{u_{i}}\right)}{U^{2} G}, \\
& H^{G}\left(\partial_{u_{i}}, \partial_{u_{j}}\right)=0, \\
& \kappa_{2}^{2}=K\left(\partial_{v_{\beta}}, \partial_{v_{\gamma}}\right)=1-\frac{|\nabla G|^{2}}{G^{2}}<1,
\end{aligned}
$$

for $1 \leq i \neq j \leq p ; p+1 \leq \beta \neq \gamma \leq n$, where $K(X, Y)$ denotes the sectional curvature of the plane section spanned by $X, Y$. Notice that (6.26) follows from (2.9), (6.23) and $\left\langle R\left(\partial_{u_{i}}, \partial_{v_{\beta}}\right) \partial_{u_{j}}, \partial_{v_{\beta}}\right\rangle=0$ with $i \neq j$.

It follows from (6.24) that $\kappa_{1}= \pm 1$. Without loss of generality, we may put $\kappa_{1}=1$. Thus we find from (6.2) and (6.22) that

$$
h\left(\partial_{u_{i}}, \partial_{u_{j}}\right)=\delta_{i j} U^{2} N, \quad h\left(\partial_{v_{\beta}}, \partial_{v_{\gamma}}\right)=\delta_{\beta \gamma} \kappa_{2} G^{2} V^{2} N, \quad h\left(\partial_{u_{i}}, \partial_{v_{\beta}}\right)=0,
$$

for $i, j=1, \ldots, p ; \beta, \gamma=p+1, \ldots, n$. Thus, by applying (6.13), (6.23), (6.28) and $\left(\bar{\nabla}_{\partial_{u_{i}}} h\right)\left(\partial_{v_{\beta}}, \partial_{v_{\beta}}\right)=\left(\bar{\nabla}_{\partial_{v_{\beta}}} h\right)\left(\partial_{u_{i}}, \partial_{v_{\beta}}\right)$, we find

$$
\frac{\partial \kappa_{2}}{\partial u_{i}}=\left(1-\kappa_{2}\right) \frac{G_{i}}{G}, \quad i=1, \ldots, p .
$$

By integrating (6.29) we obtain

$$
\kappa_{2}=1-\frac{c}{G}
$$

for some constant $c \neq 0$. Therefore (6.25), (6.27) and (6.30) yield

$$
\begin{aligned}
& H^{G}\left(e_{i}, e_{j}\right)=\delta_{i j}(c-G), \\
& |\nabla G|^{2}=c(2 G-c),
\end{aligned}
$$

for any orthonormal basis $\left\{e_{1}, \ldots, e_{p}\right\}$ of $\operatorname{Span}\left\{\partial_{u_{1}}, \ldots, \partial_{u_{p}}\right\}$. 
To solve the PDE system (6.31)-66.32), we apply a spherical coordinate system $\left\{x_{1}, \ldots, x_{p}\right\}$ for the first factor $S^{p}(1)$ of the warped product $S^{p}(1) \times_{G} S^{n-p}(1)$ so that the metric tensor $g_{1}$ on $S^{p}(1)$ is given by

$$
g_{1}=d x_{1}^{2}+\cos ^{2} x_{1} d x_{2}^{2}+\cdots+\prod_{k=1}^{p-1} \cos ^{2} x_{k} d x_{p}^{2} .
$$

The Levi-Civita connection $\stackrel{\circ}{\nabla}$ of $g_{1}$ satisfies

$$
\begin{aligned}
\stackrel{\circ}{\nabla}_{\partial_{x_{1}}} \partial_{x_{1}}= & 0, \\
\stackrel{\circ}{\nabla}_{\partial_{x_{i}}} \partial_{x_{j}}= & -\left(\tan x_{i}\right) \partial_{x_{j}}, \quad 1 \leq i<j, \\
\stackrel{\circ}{\nabla}_{\partial_{x_{2}}} \partial_{x_{2}}= & \frac{\sin 2 x_{1}}{2} \partial_{x_{1}}, \\
& \ldots \\
\stackrel{\circ}{\nabla}_{\partial_{x_{p}}} \partial_{x_{p}}= & \sum_{k=1}^{p-1}\left(\frac{\sin 2 x_{k}}{2} \prod_{\ell=k+1}^{p-1} \cos ^{2} x_{\ell}\right) \partial_{x_{k}} .
\end{aligned}
$$

It follows from (6.31) and (6.33) that

$$
H^{G}\left(\partial_{x_{i}}, \partial_{x_{j}}\right)=\delta_{i j}(c-G)\left(\prod_{k=1}^{i-1} \cos x_{k}\right)\left(\prod_{\ell=1}^{j-1} \cos x_{\ell}\right),
$$

In particular, for $i=j=1$, we find from (2.9), (6.34) and (6.35) that $G_{x_{1} x_{1}}=c-G$, which gives

$$
G=c+A_{0}\left(x_{2}, \ldots, x_{p}\right) \cos x_{1}+B_{0}\left(x_{2}, \ldots, x_{p}\right) \sin x_{1}
$$

for some functions $A_{0}\left(x_{2}, \ldots, x_{p}\right)$ and $B_{0}\left(x_{2}, \ldots, x_{p}\right)$.

For $i=1$ and $j=2, \ldots, p$, we derive from (2.9), (6.34) and (6.35) that

$$
0=H^{G}\left(\partial_{x_{1}}, \partial_{x_{j}}\right)=G_{x_{1} x_{j}}-\tan x_{1} G_{x_{j}} .
$$

By substituting (6.36) into (6.37) we obtain $\partial B_{0} / \partial x_{j}=0$ for $j=2, \ldots, p$. Thus $B_{0}$ is a constant, say $c_{1}$. So (6.36) becomes

$$
G=c+A_{0}\left(x_{2}, \ldots, x_{p}\right) \cos x_{1}+c_{1} \sin x_{1} .
$$

Similarly, by substituting (6.38) into (6.35) for $i=j=2$ and applying (2.9) and (6.34), we obtain

$$
\begin{aligned}
G=c+ & c_{1} \sin x_{1}+A_{1}\left(x_{3}, \ldots, x_{p}\right) \cos x_{1} \cos x_{2} \\
& +B_{1}\left(x_{3}, \ldots, x_{p}\right) \cos x_{1} \sin x_{2}
\end{aligned}
$$

for some functions $A_{1}\left(x_{3}, \ldots, x_{p}\right), B_{1}\left(x_{3}, \ldots, x_{p}\right)$. Continuing such procedures for sufficient many times, we arrive that

$$
G=c+c_{1} \sin x_{1}+\cdots+c_{p-1} \sin x_{p-1} \prod_{j=1}^{p-2} \cos x_{j}+c_{p} \prod_{j=1}^{p} \cos x_{j},
$$


where $c, c_{1}, \ldots, c_{p}$ are real numbers, not all zero. On the other hand, by substituting (6.40) into (6.32), we find $c=c_{1}=\cdots=c_{p}=0$, which is a contradiction. Hence this case is impossible.

Case (2.2): $p=1$ or $n-1$. Without loss of generality, we may assume $p=1$. Now, we divide this into two cases.

Case (2.2.1): $n=2$. In this case, we have $\operatorname{Ric}(X, Y)=\tau g(X, Y), X, Y \in T M^{2}$, where $\tau$ is the Gauss curvature of $M^{2}$. Hence (4.1) of Theorem 4.1 gives

$$
\tilde{g}\left(h(X, Y), \mathbf{x}^{\perp}\right)=(\lambda-1-\tau) g(X, Y) .
$$

Since $M^{2}$ is assumed to have two distinct principal curvatures, 6.41) implies that $\mathbf{x}^{\perp}=0$. Hence $\mathbf{x}$ must be tangent to $M^{2}$. So, it follows from $\tilde{\nabla}_{X} \mathbf{x}=X$ that the second fundamental form satisfies $h(\mathbf{x}, X)=0$ for any $X \in T M^{2}$. Thus at least one $\kappa_{1}, \kappa_{2}$ is zero, which is a contradiction.

Case (2.2.2): $n \geq 3$. In this case, we have $m\left(\kappa_{1}\right)=1, m\left(\kappa_{2}\right)=n-1 \geq 2$. Moreover, (6.2) reduces to

$$
\begin{gathered}
h\left(e_{1}, e_{1}\right)=\kappa_{1} N, \quad h\left(e_{i}, e_{\beta}\right)=0, h\left(e_{\beta}, e_{\gamma}\right)=\delta_{\beta \gamma} \kappa_{2} N, \\
\beta, \gamma=2, \ldots, n,
\end{gathered}
$$

with respect to an orthonormal tangent frame $\left\{e_{1}, \ldots, e_{n}\right\}$ of $M^{n}$

From (2.7), (6.42) and $\left(\bar{\nabla}_{e_{\alpha}} h\right)\left(e_{\beta}, e_{\gamma}\right)=\left(\bar{\nabla}_{e_{\beta}} h\right)\left(e_{\alpha}, e_{\gamma}\right), 2 \leq \beta \neq \gamma \leq n$, we find $e_{\beta} \kappa_{2}=0$ for $\beta=2, \ldots, n$. So we get

$$
\nabla \kappa_{2}=f e_{1} \in \mathcal{D}_{1}
$$

for some function $f$. Also, (2.7), (6.42) and $\left(\bar{\nabla}_{e_{1}} h\right)\left(e_{\beta}, e_{\gamma}\right)=\left(\bar{\nabla}_{e_{\beta}} h\right)\left(e_{1}, e_{\gamma}\right)$ give

$$
\omega_{\beta}^{1}\left(e_{\gamma}\right)=\delta_{\beta \gamma}\left(\frac{e_{1} \kappa_{2}}{\kappa_{2}-\kappa_{1}}\right), \beta, \gamma=2, \ldots, n .
$$

From (6.44) we find $\left\langle\left[e_{i}, e_{j}\right], e_{1}\right\rangle=0$. Thus $\mathcal{D}_{2}$ is integrable. Also, it follows from (6.44) that the second fundamental form $\stackrel{\circ}{h}$ of a leaf $F^{n-1}$ of $\mathcal{D}_{2}$ in $M^{n}$ is given by

$$
\stackrel{\circ}{h}\left(e_{\beta}, e_{\gamma}\right)=\delta_{\beta \gamma}\left(\frac{e_{1} \kappa_{2}}{\kappa_{2}-\kappa_{1}}\right) e_{1}, \beta, \gamma=2, \ldots, n .
$$

Hence $F^{n-1}$ is a totally umbilical hypersurface of $M^{n}$. It follows from (6.42) and (6.45) that the second fundamental form $\hat{h}$ and the mean curvature vector $\hat{H}$ of $F^{n-1}$ in $\mathbb{E}^{n+1}$ are given respectively by

$$
\begin{aligned}
& \hat{h}\left(e_{\beta}, e_{\gamma}\right)=\delta_{\beta \gamma}\left\{\left(\frac{e_{1} \kappa_{2}}{\kappa_{2}-\kappa_{1}}\right) e_{1}+\kappa_{2} N\right\}, \\
& \hat{H}=\stackrel{\circ}{H}+\kappa_{2} N, \stackrel{\circ}{H}=\left(\frac{e_{1} \kappa_{2}}{\kappa_{2}-\kappa_{1}}\right) e_{1} .
\end{aligned}
$$

From (6.46) we see that $F^{n-1}$ is also totally umbilical in $\mathbb{E}^{n+1}$. Hence $F^{n-1}$ is an open portion of an $(n-1)$-sphere $S^{n-1}$. So the mean curvature vector $\hat{H}$ of $F^{n-1}$ is parallel in the normal bundle in $\mathbb{E}^{n+1}$, i.e., $\hat{D}_{X} \hat{H}=0$ for $X \in T F^{n-1}$, where $\hat{D}$ 
is the normal connection of $F^{n-1}$ in $\mathbb{E}^{n+1}$. Now, by applying Weingarten's formula for $F^{n-1}$ in $\mathbb{E}^{n+1}$, we find

$$
\tilde{\nabla}_{e_{\beta}} \hat{H}=-\hat{A}_{\hat{H}} e_{\beta}=\eta e_{\beta}
$$

for some function $\eta$, where $\hat{A}$ denotes the shape operator of $F^{n-1}$ in $\mathbb{E}^{n+1}$. On the other hand, we find from (6.47) and formulas of Gauss and Weingarten that

$$
\tilde{\nabla}_{e_{\beta}} \hat{H}=-{\stackrel{\circ}{\AA_{H}}}_{\iota_{\beta}}+\stackrel{\circ}{D}_{e_{\beta}} \stackrel{\circ}{H}+h\left(e_{\beta}, \stackrel{\circ}{H}\right)-\kappa_{2}^{2} e_{\beta}+\left(e_{\beta} \kappa_{2}\right) N
$$

for $\beta=2, \ldots, n$, where $\AA$ and $\stackrel{\circ}{D}$ are the shape operator and the normal connection of $F^{n-1}$ in $M^{n}$, respectively. Thus, as case (2.1), $F^{n-1}$ has parallel mean curvature vector in the normal bundle in $M^{n}$. Consequently, $\mathcal{D}_{2}$ is a spherical distribution.

On the other hand, because $\mathcal{D}_{1}$ is of rank one, $\mathcal{D}_{1}$ is integrable and its leaves are clearly totally umbilical in $M^{n}$. Therefore, by Theorem [3.2, $M^{n}$ is locally a twisted-warped product $I \times{ }_{(P, Q)} S^{n-1}(1)$ whose metric tensor is

$$
g=P^{2}\left(s, y_{2}, \ldots, y_{n}\right) d s^{2}+f^{2}(s) g_{S^{n-1}}
$$

where $I$ is an open interval with arclength $s, P$ is function on $M^{n}$ and $g_{S^{n-1}}$ is the metric tensors of $S^{n-1}(1)$. In terms of a spherical coordinate system $\left\{y_{2}, \ldots, y_{n}\right\}$ of $S^{n-1}(1)$, (6.50) can be expressed as

$$
g=P^{2} d s^{2}+f(s)^{2}\left\{d y_{2}^{2}+\cos ^{2} y_{2} d y_{3}^{2}+\cdots+\prod_{k=2}^{n-1} \cos ^{2} y_{k} d y_{n}^{2}\right\} .
$$

Hence the Levi-Civita connection $\nabla$ of $M^{n}$ satisfies

$$
\begin{aligned}
& \nabla_{\partial_{s}} \partial_{s}=\frac{P_{s}}{P} \partial_{s}-\frac{P}{f^{2}(s)}\left\{P_{y_{2}} \partial_{y_{2}}+\sum_{\alpha=3}^{n}\left(\sec ^{2} y_{2} \cdots \sec ^{2} y_{\alpha-1}\right) P_{y_{\alpha}} \partial_{y_{\alpha}}\right\} \\
& \nabla_{\partial_{s}} \partial_{y_{\beta}}=\frac{P_{y_{\beta}}}{P} \partial_{s}+\frac{f^{\prime}}{f} \partial_{y_{\beta}}, \quad 2 \leq \beta \leq n \\
& \nabla_{\partial_{y_{2}}} \partial_{y_{2}}=-\frac{f f^{\prime}}{P^{2}} \partial_{s} \\
& \nabla_{\partial_{y_{3}}} \partial_{y_{3}}=-\frac{f f^{\prime}}{P^{2}}\left(\cos ^{2} y_{2}\right) \partial_{s}+\frac{\sin 2 y_{2}}{2} \partial_{y_{2}}, \\
& \quad \quad \ldots \\
& \nabla_{\partial_{y_{n}}} \partial_{y_{n}}=-\frac{f f^{\prime}}{P^{2}}\left\{\prod_{\alpha=2}^{n-1} \cos ^{2} y_{\alpha}\right\} \partial_{s}+\sum_{\alpha=2}^{n-1}\left\{\frac{\sin 2 y_{\alpha}}{2} \prod_{\gamma=\alpha+1}^{n-1} \cos ^{2} y_{\gamma}\right\} \partial_{y_{\alpha}} \\
& \nabla_{\partial_{y_{\beta}}} \partial_{y_{\gamma}}=-\left(\tan y_{\beta}\right) \partial_{y_{\gamma}}, 2 \leq \beta<\gamma \leq n .
\end{aligned}
$$

By (6.42), (6.52) and Gauss' equation, we derive from $0=g\left(R\left(\partial_{s}, \partial_{y_{\beta}}\right) \partial_{y_{\gamma}}, \partial_{y_{\beta}}\right)$ that $f^{\prime}(s) P_{y_{\beta}}=0$ for $\beta=2, \ldots, n$. Consequently, we have
(a) $P=P(s)$ or
(b) $f$ is a constant, say $b \neq 0$. 
Case (2.2.2.a): $P=P(s)$. In this case, $M^{n}$ is an ordinary warped product whose metric tensor is

$$
g=P^{2}(s) d s^{2}+f(s)^{2}\left(d y_{2}^{2}+\cos ^{2} y_{2} d y_{3}^{2}+\cdots+\prod_{\alpha=2}^{n-1} \cos ^{2} y_{\alpha} d y_{n}^{2}\right) .
$$

Hence the Levi-Civita connection $\nabla$ of $M^{n}$ satisfies

$$
\begin{aligned}
\nabla_{\partial_{s}} \partial_{s}= & \frac{P_{s}}{P} \partial_{s}, \\
\nabla_{\partial_{s}} \partial_{y_{\beta}} & =\frac{f^{\prime}}{f} \partial_{y_{\beta}}, \quad 2 \leq \beta \leq n, \\
\nabla_{\partial_{y_{2}}} \partial_{y_{2}} & =-\frac{f f^{\prime}}{P^{2}} \partial_{s}, \\
& \ldots \\
\nabla_{\partial_{y_{n}}} \partial_{y_{n}} & =-\frac{f f^{\prime}}{P^{2}}\left\{\prod_{\alpha=2}^{n-1} \cos ^{2} y_{\alpha}\right\} \partial_{s}+\sum_{\alpha=2}^{n-1}\left\{\frac{\sin 2 y_{\alpha}}{2} \prod_{\gamma=\alpha+1}^{n-1} \cos ^{2} y_{\gamma}\right\} \partial_{y_{\alpha}}, \\
\nabla_{\partial_{y_{\beta}}} \partial_{y_{\gamma}} & =-\left(\tan y_{\beta}\right) \partial_{y_{\gamma}}, 2 \leq \beta<\gamma \leq n .
\end{aligned}
$$

By applying (6.54) and a direct computation, we find

$$
\begin{aligned}
& K\left(\partial_{s}, \partial_{y_{\beta}}\right)=\frac{f^{\prime}(s) P^{\prime}(s)-P(s) f^{\prime \prime}(s)}{f(s) P(s)^{3}} \\
& K\left(\partial_{y_{\beta}}, \partial_{y_{\gamma}}\right)=\frac{P^{2}(s)-f^{\prime}(s)^{2}}{f^{2}(s) P^{2}(s)}, \quad 2 \leq \beta, \gamma \leq n .
\end{aligned}
$$

On the other hand, it follows from (6.42) and the equation of Gauss that

$$
K\left(\partial_{s}, \partial_{y_{\beta}}\right)=\kappa_{1} \kappa_{2}, \quad K\left(\partial_{y_{\beta}}, \partial_{y_{\gamma}}\right)=\kappa_{2}^{2}, \quad 2 \leq \beta \neq \gamma \leq n
$$

Thus we derive from (6.55) and (6.56) that

$$
\kappa_{1} \kappa_{2}=\frac{f^{\prime}(s) P^{\prime}(s)-P(s) f^{\prime \prime}(s)}{f(s) P(s)^{3}}, \kappa_{2}^{2}=\frac{P^{2}(s)-f^{\prime}(s)^{2}}{f^{2}(s) P^{2}(s)},
$$

which imply that $\kappa_{1}=\kappa_{1}(s)$ and $\kappa_{2}=\kappa_{2}(s)$.

Case (2.2.2.a.i): $P(s)>f^{\prime}(s)$. We may put

$$
\kappa_{2}=\frac{\sqrt{P^{2}(s)-f^{\prime}(s)^{2}}}{f(s) P(s)},
$$

From (6.57) and (6.58) we get

$$
\kappa_{1}=\frac{f^{\prime} P^{\prime}-P f^{\prime \prime}}{P^{2} \sqrt{P^{2}(s)-f^{\prime}(s)^{2}}} .
$$


Let $L: M^{n} \rightarrow \mathbb{E}^{n+1}$ denote the isometric immersion of $M^{n}$ in $\mathbb{E}^{n+1}$. We derive from (6.42), (6.53), (6.54), (6.58), (6.59) and formula (2.1) of Gauss that

$$
\begin{aligned}
L_{s s}= & \frac{P^{\prime}}{P} L_{s}+\frac{f^{\prime} P^{\prime}-P f^{\prime \prime}}{\sqrt{P^{2}-f^{\prime 2}}} N, \\
L_{s y_{\beta}}= & \frac{f^{\prime}}{f} L_{y_{\beta}}, \quad \beta=2, \ldots, n, \\
L_{y_{2} y_{2}}= & -\frac{f f^{\prime}}{P^{2}} \partial_{s}+\frac{f \sqrt{P^{2}-f^{\prime 2}}}{P} N, \\
L_{y_{\beta} y_{\beta}}= & -\frac{f f^{\prime}}{P^{2}}\left\{\prod_{\alpha=2}^{\beta-1} \cos ^{2} y_{\alpha}\right\} L_{s}+\sum_{\alpha=2}^{\beta-1}\left\{\frac{\sin 2 y_{\alpha}}{2} \prod_{\gamma=\alpha+1}^{\beta-1} \cos ^{2} y_{\gamma}\right\} L_{y_{\alpha}} \\
& +\frac{f \sqrt{P^{2}-f^{\prime 2}}}{P}\left(\prod_{\alpha=2}^{\beta-1} \cos ^{2} y_{\alpha}\right) N, \beta=3, \ldots, n, \\
L_{y_{\beta} y_{\gamma}}= & -\left(\tan y_{\beta}\right) L_{y_{\gamma}}, \quad 2 \leq \beta<\gamma \leq n .
\end{aligned}
$$

From (6.58), (6.59) and formula of Weingarten we also have

$$
N_{s}=\frac{f^{\prime} P^{\prime}-P f^{\prime \prime}}{P^{2} \sqrt{P^{2}-f^{\prime 2}}} L_{s}, \quad N_{y_{\beta}}=\frac{\sqrt{P^{2}-f^{\prime 2}}}{f P} L_{y_{\beta}}, \quad \beta=2, \ldots, n .
$$

After solving equation (6.61) for $\beta=2, \ldots, n$, we find

$$
L\left(s, y_{2}, \ldots, y_{n}\right)=A(s)+f(s) B\left(y_{2}, \ldots, y_{n}\right)
$$

for $\mathbb{E}^{n+1}$-valued functions $A(s)$ and $B\left(y_{2}, \ldots, y_{n}\right)$. By applying (6.66), we conclude after a long computation that the solution of the PDE system (6.60)-(6.65) is

$$
\begin{gathered}
L=\mathbf{c}_{0} \int^{s} \sqrt{P^{2}(t)-f^{\prime}(t)^{2}} d t+f(x)\left\{\mathbf{c}_{1} \sin y_{2}+\mathbf{c}_{2} \cos y_{2} \sin y_{3}+\cdots\right. \\
\left.+\mathbf{c}_{n-1} \sin y_{n-1} \prod_{\alpha=2}^{n-2} \cos y_{n-1}+\mathbf{c}_{n} \prod_{\alpha=2}^{n-1} \cos y_{\alpha}\right\}
\end{gathered}
$$

for some vectors $\mathbf{c}_{0}, \ldots, \mathbf{c}_{n} \in \mathbb{E}^{n+1}$. From (6.53) and (6.67) we know that $\mathbf{c}_{0}, \ldots, \mathbf{c}_{n}$ are orthonormal. Therefore the immersion of $M^{n}$ is congruent to

$$
\begin{gathered}
L=\left(\int s \sqrt{P^{2}(t)-f^{\prime}(t)^{2}} d t, f(x) \sin y_{2}, f(s) \cos y_{2} \sin y_{3}, \cdots\right. \\
\left.f(s) \sin y_{n-1} \prod_{\alpha=2}^{n-2} \cos y_{n-1}, f(s) \prod_{\alpha=2}^{n-1} \cos y_{\alpha}\right)
\end{gathered}
$$

Now, by applying a suitable reparametrization of $s$, we conclude that $L$ takes the form of (4.3) in Lemma 4.1. Consequently, after applying Lemma 4.1, we know that $M^{n}$ is an open part of a hypersphere, which is a contradiction. 
Case (2.2.2.a.ii): $P(s)<f^{\prime}(s)$. In this case, by (6.57), we may put

$$
\kappa_{2}=\frac{\sqrt{f^{\prime}(s)^{2}-P^{2}(s)}}{f(s) P(s)}, \quad \kappa_{1}=\frac{f^{\prime} P^{\prime}-P f^{\prime \prime}}{P^{2} \sqrt{f^{\prime}(s)^{2}-P^{2}(s)}} .
$$

We derive from (6.42), (6.54), (6.68) and

$$
\left(\bar{\nabla}_{\partial_{s}} h\right)\left(\partial_{y_{2}}, \partial_{y_{2}}\right)=\left(\bar{\nabla}_{\partial_{y_{2}}} h\right)\left(\partial_{s}, \partial_{y_{2}}\right)
$$

of Codazzi that $f^{\prime}\left(f^{\prime} P^{\prime}-P f^{\prime \prime}\right)=0$. Since $\kappa_{1}, \kappa_{2} \neq 0$, (6.68) implies $f^{\prime} P^{\prime}-P f^{\prime \prime} \neq 0$. So we get $f^{\prime}=0$, which is impossible by (6.68) since $\kappa_{2}$ is real. Consequently, this case is also impossible.

Case (2.2.2.b): $f$ is a constant $b \neq 0$. After applying a suitable dilation, we get $b=1$. Thus $M^{n}$ is an ordinary twisted product whose metric tensor is

$$
g=P^{2}\left(s, y_{2}, \ldots, y_{n}\right) d s^{2}+\left\{d y_{2}^{2}+\cos ^{2} y_{2} d y_{3}^{2}+\cdots+\prod_{k=2}^{n-1} \cos ^{2} y_{k} d y_{n}^{2}\right\} .
$$

Hence the Levi-Civita connection $\nabla$ of $M^{n}$ satisfies

$$
\begin{aligned}
& \nabla_{\partial_{s}} \partial_{s}= \frac{P_{s}}{P} \partial_{s}-P^{2}\left\{P_{y_{2}} \partial_{y_{2}}+\sum_{\alpha=3}^{n}\left(\sec ^{2} y_{2} \cdots \sec ^{2} y_{\alpha-1}\right) \partial_{y_{\alpha}}\right\} \\
& \nabla_{\partial_{s}} \partial_{y_{\beta}}= \frac{P_{y_{\beta}}}{P} \partial_{s}, \quad 2 \leq \beta \leq n \\
& \nabla_{\partial_{y_{2}}} \partial_{y_{2}}= 0, \quad \nabla_{\partial_{y_{3}}} \partial_{y_{3}}=\frac{\sin 2 y_{2}}{2} \partial_{y_{2}} \\
& \cdots \\
& \nabla_{\partial_{y_{n}}} \partial_{y_{n}}=\sum_{\alpha=2}^{n-1}\left\{\frac{\sin 2 y_{\alpha}}{2} \prod_{\gamma=\alpha+1}^{n-1} \cos ^{2} y_{\gamma}\right\} \partial_{y_{\alpha}} \\
& \nabla_{\partial_{y_{\beta}} \partial_{y_{\gamma}}}=-\left(\tan y_{\beta}\right) \partial_{y_{\gamma}}, \quad 2 \leq \beta<\gamma \leq n .
\end{aligned}
$$

It follows from (6.42), (6.70) and $0=\left\langle R\left(\partial_{s}, \partial_{y_{\beta}}\right) \partial_{y_{\gamma}}, \partial_{s}\right\rangle, \beta<\gamma$, that

$$
P_{y_{\beta} y_{\gamma}}+\left(\tan y_{\beta}\right) P_{y_{\gamma}}=0,2 \leq \beta<\gamma \leq n .
$$

After solving (6.71) we obtain

$$
P=A_{1}\left(s, y_{2}\right)+\left(\cos y_{2}\right) A_{2}\left(s, y_{3}\right)+\cdots+\left(\prod_{\alpha=2}^{n-1} \cos y_{\alpha}\right) A_{n-1}\left(s, y_{n}\right) .
$$

Also, it follows from (6.69) that

$$
K\left(\partial_{s}, \partial_{y_{\beta}}\right)=-\frac{P_{y_{\beta} y_{\beta}}}{P}, \quad K\left(\partial_{y_{\beta}}, \partial_{y_{\gamma}}\right)=1,2 \leq \beta<\gamma \leq n .
$$

Thus we get

$$
\kappa_{1}=-\frac{P_{y_{\beta} y_{\beta}}}{P}, \quad \kappa_{2}=1 .
$$


From the first equation in (6.74) we find

$$
P_{y_{2} y_{2}}=\cdots=P_{y_{n} y_{n}} .
$$

From (6.72) and 6.75) we have

$$
P=A(s)+\cos y_{2} \cdots \cos y_{n-1}\left\{C(s) \sin y_{n}+D(s) \cos y_{n}\right\} .
$$

After applying a suitable translation in $y_{n}$ and a reparametrization of $s$, (6.76) reduces to $P=A(s)+\prod_{\alpha=2}^{n} \cos y_{\alpha}$. Hence (6.69) becomes

$$
g=\left(A(s)+\prod_{\alpha=2}^{n} \cos y_{\alpha}\right)^{2} d s^{2}+\left\{d y_{2}^{2}+\cos ^{2} y_{2} d y_{3}^{2}+\cdots+\prod_{k=2}^{n-1} \cos ^{2} y_{k} d y_{n}^{2}\right\}
$$

It follows from (6.74) and (6.77) that

$$
\kappa_{1}=\frac{\prod_{\alpha=2}^{n} \cos y_{\alpha}}{A(s)+\prod_{\alpha=2}^{n} \cos y_{\alpha}}, \quad \kappa_{2}=1 .
$$

Let $L: M^{n} \rightarrow \mathbb{E}^{n+1}$ be the isometric immersion of $M^{n}$ in $\mathbb{E}^{n+1}$. We derive from (6.42), (6.69), (6.70), (6.72), (6.76), (6.78) and formula (2.1) of Gauss that

$$
\begin{aligned}
& L_{s s}=\frac{A^{\prime}}{A} L_{s}-\left(A(s)+\prod_{\alpha=2}^{n} \cos y_{\alpha}\right)^{2} \sum_{\beta=2}^{n}\left(\sec ^{2} y_{2} \cdots \sec ^{2} y_{\beta-1}\right) L_{y_{\beta}} \\
& \quad-\left(A(s)+\prod_{\alpha=2}^{n} \cos y_{\alpha}\right) \prod_{\beta=2}^{n} \cos y_{\beta} N, \\
& L_{s y_{\beta}}=\frac{-\sin y_{\beta} \prod_{\alpha \neq \beta} \cos y_{\alpha}}{A(s)+\prod_{\alpha=2}^{n} \cos y_{\alpha}} L_{s}, \beta=2, \ldots, n, \\
& L_{y_{2} y_{2}}=N, \quad \frac{\sin 2 y_{2}}{2} L_{y_{2}}+\cos ^{2} y_{2} N, \\
& L y_{3} y_{3}=\frac{\ldots}{\cdots} \\
& L_{y_{n} y_{n}}=\sum_{\alpha=2}^{n-1}\left\{\frac{\sin 2 y_{\alpha}}{2} \prod_{\gamma=\alpha+1}^{n-1} \cos ^{2} y_{\gamma}\right\} L_{y_{\alpha}}+\left(\prod_{\alpha=2}^{n-1} \cos ^{2} y_{\alpha}\right) N, \\
& L_{y_{\beta} y_{\gamma}}=-\left(\tan y_{\beta}\right) L_{y_{\gamma}}, \quad 2 \leq \beta<\gamma \leq n .
\end{aligned}
$$

Moreover, from (6.78) and formula of Weingarten we also have

$$
N_{s}=-\frac{P_{y_{\beta} y_{\beta}}}{P} L_{s}, \quad N_{y_{\beta}}=L_{y_{\beta}}, \quad \beta=2, \ldots, n .
$$

We obtain by solving the PDE system (6.79)-(6.80) after very long computation that

$$
\begin{array}{r}
L\left(s, y_{2}, \ldots, y_{n}\right)=\int^{s} A(t)\left(\mathbf{c}_{1} \cos t-\mathbf{c}_{2} \sin t\right) d t+\mathbf{c}_{3} \sin y_{2}+\cdots \\
\quad+\mathbf{c}_{n+1} \sin y_{n} \prod_{\alpha=2}^{n-1} \cos y_{\alpha}+\left(\mathbf{c}_{1} \cos t+\mathbf{c}_{2} \sin t\right)\left(\prod_{\alpha=2}^{n} \cos y_{\alpha}\right)
\end{array}
$$


for some vectors $\mathbf{c}_{1}, \ldots, \mathbf{c}_{n+1} \in \mathbb{E}^{n+1}$. It follows from (6.69), (6.77) and (6.81) that $\mathbf{c}_{1}, \ldots, \mathbf{c}_{n+1}$ are orthonormal vectors. Hence, after choosing

$$
\mathbf{c}_{1}=(1,0, \ldots, 0), \ldots, \mathbf{c}_{n+1}=(0, \ldots, 0,1)
$$

we obtain from 6.81) that

$$
\begin{gathered}
L=\left(\cos s \prod_{\alpha=2}^{n} \cos y_{\alpha}+\int^{s} A(t) \cos t d t, \sin s \prod_{\alpha=2}^{n} \cos y_{\alpha}-\int^{s} A(t) \sin t d t\right. \\
\left.\sin y_{2}, \cdots, \sin y_{n} \prod_{\alpha=2}^{n-1} \cos y_{\alpha}\right)
\end{gathered}
$$

From (6.77) we find

$$
\operatorname{Ric}\left(\partial_{y_{2}}, \partial_{y_{2}}\right)=1+\frac{\prod_{\beta=2}^{n} \cos y_{\beta}}{A(s)+\prod_{\alpha=2}^{n} \cos y_{\alpha}}
$$

On the other hand, we derive from (6.77) and (6.82) that the second fundamental form $h$ of $M^{n}$ in $\mathbb{E}^{n+1}$ satisfies

$$
h\left(\partial_{y_{2}}, \partial_{y_{2}}\right)=-\left(\cos s \prod_{\alpha=2}^{n} \cos y_{\alpha}, \sin s \prod_{\alpha=2}^{n} \cos y_{\alpha}, \sin y_{2}, \cdots, \sin y_{n} \prod_{\alpha=2}^{n-1} \cos y_{\alpha}\right)
$$

Now, it is easy to verify that

$$
\frac{\operatorname{Ric}\left(\partial_{y_{2}}, \partial_{y_{2}}\right)-\left\langle h\left(\partial_{y_{2}}, \partial_{y_{2}}\right), L\right\rangle}{g_{22}}
$$

cannot be equal to $\lambda-1$ for any constant $\lambda$. Consequently, $\left(M^{n}, g, \mathbf{x}^{T}, \lambda\right)$ is not a Ricci soliton for any $\lambda$. Hence this case is impossible as well.

From the proof of Theorem 6.1 we obtain the following.

Corollary 6.1. There do not exist steady or expanding Ricci solitons $\left(M^{n}, g, \mathbf{x}^{T}, \lambda\right)$ on Euclidean hypersurfaces.

Acknowledgements. This work is supported by NPST program of King Saud University Project Number 13-MAT1813-02. Both authors thank the referee for his/her very careful reading of this paper and for providing useful suggestions for improving the presentation of this paper.

\section{REFERENCES}

[1] H. Al-Sodais, H. Alodan and S. Deshmukh, Hypersurfaces of Euclidean space as gradient Ricci solitons, An. Ştiinţ. Univ. Al. I. Cuza Iaşi. Mat. (N.S.) DOI:10.2478/aicu-2014-0009.

[2] B.-Y. Chen, Geometry of Submanifolds, Pure and Applied Mathematics, No. 22, Mercer Dekker Inc., New York, 1973.

[3] B.-Y. Chen, Geometry of Submanifolds and Its Application, Science University of Tokyo, Tokyo, Japan, 1981.

[4] B.-Y. Chen, Geometry of position functions of Riemannian submanifolds in pseudo-Euclidean space, J. Geom. 74 (2002), no. 1-2, 61-77. 
[5] B.-Y. Chen, Constant-ratio space-like submanifolds in pseudo-Euclidean space, Houston J. Math. 29 (2003), no. 2, 281-294

[6] B.-Y. Chen, Pseudo-Riemannian Geometry, $\delta$-invariants and Applications, World Scientific, Hackensack, NJ, 2011.

[7] B.-Y. Chen, Total Mean Curvature and Submanifolds of Finite Type, Second Edition, World Scientific, Hackensack, NJ, 2014.

[8] B.-Y. Chen and S. Deshmukh, Geometry of compact shrinking Ricci solitons, Balkan J. Geom. Appl. 19 (2014), no. 1, 13-21.

[9] B.-Y. Chen and S. Deshmukh, Ricci solitons and concurrent vector fields, arXiv:1407.2790, 2014.

[10] B.-Y. Chen and K. Yano, On submanifolds of submanifolds of a Riemannian manifold, J. Math. Soc. Japan, 23 (1971), no. 3, 548-554.

[11] J. T. Cho and M. Kimura, Ricci solitons and real hypersurfaces in a complex space form, Tohoku Math. J. (2) 61 (2009), no. 2, 205-212.

[12] J. T. Cho and M. Kimura, Ricci solitons of compact real hypersurfaces in Kähler manifolds, Math. Nachr. 284 (2011), no. 11-12, 1385-1393.

[13] J. T. Cho and M. Kimura, Ricci solitons on locally conformally flat hypersurfaces in space forms, J. Geom. Phys. 62 (2012), no. 8, 1882-1891.

[14] A. Derdzinski, A Myers-type theorem and compact Ricci solitons, Proc. Amer. Math. Soc. 134 (2006), no. 12, 3645-3648.

[15] S. Deshmukh, Jacobi-type vector fields on Ricci solitons, Bull. Math. Soc. Sci. Math. Roumanie 55(103) (2012), no. 1, 41-50.

[16] R. S. Hamilton, The formation of singularities in the Ricci flow, In: Surveys in Differential Geometry, Vol. II, (1995), 7-136.

[17] S. Hiepko, Eine innere Kennzeichnung der verzerrten Produkte, Math. Ann. 241 (1979), no. 3, 209-215.

[18] M. F. Lopéz and E. García-Río, A remark on compact Ricci solitons, Math. Ann. 340 (2008), no. 4, 893-896.

[19] J. Morgan and G. Tian, Ricci Flow and the Poincaré Conjecture, Clay Mathematics Monographs, Vol. 5, Cambridge, MA, 2014.

[20] G. Perelman, The Entropy Formula for the Ricci Flow and Its Geometric Applications, 2002, Zbl 1130.53001; arXiv math/0211159

[21] R. Ponge and H. Reckziegel, Twisted products in pseudo-Riemannian geometry, Geom. Dedicata, 48 (1993), no. 1, 15-25.

[22] B. Ünal, Doubly warped products, Differential Geom. Appl. 15 (2001), no. 3, 253-263.

Department of Mathematics, Michigan State University, 619 Red Cedar Road, East LANSING, MI 48824-1027, USA

E-mail address: bychen@math.msu.edu

Department of Mathematics, King Saud University, Riyadh 11451, Saudi Arabia

E-mail address: shariefd@ksu.edu.sa 